\title{
Stabilizing breathing pattern using local mechanical vibrations: comparison of deterministic and stochastic stimulations in rodent models of apnea of prematurity
}

\author{
Dean Zeldich ${ }^{1}$. Samer Bou Jawde ${ }^{1}$. Jacob Herrmann ${ }^{1}$. Leen Arnaout ${ }^{1} \cdot$ Meghan Griffin $^{1} \cdot$ Noam Grunfeld $^{1}$. \\ Yu Zhang ${ }^{1} \cdot$ Ramaswamy Krishnan $^{2} \cdot$ Erzsébet Bartolák-Suki $^{1} \cdot$ Béla Suki $^{1}$ (i)
}

Received: 18 May 2021 / Revised: 17 August 2021 / Accepted: 20 August 2021 / Published online: 1 September 2021

(c) Korean Society of Medical and Biological Engineering 2021

\begin{abstract}
Mechanical stimulation has been shown to reduce apnea of prematurity (AOP), a major concern in preterm infants. Previous work suggested that the underlying mechanism is stochastic resonance, amplification of a subthreshold signal by stochastic stimulation. We hypothesized that the mechanism behind the reduction of apnea length may not be a solely stochastic phenomenon, and suggest that a purely deterministic, non-random mechanical stimulation could be equally as effective. Mice and rats were anesthetized, tracheostomized, and mechanically ventilated to halt spontaneous breathing. Two miniature motors controlled by a microcontroller were attached around the abdomen. Ventilation was paused, stimulations were applied, and the time to the rodent's first spontaneous breath (T) was measured. Six spectrally different signals were compared to one another and the no-stimulation control in mice. The most successful deterministic stimulation (D) at reducing apnea was then compared to a pseudo-random noise (PRN) signal of comparable amplitude and frequency. $\mathrm{CO}_{2} \%, \mathrm{CO}_{2}$ stabilization time $\left(\mathrm{T}_{\mathrm{s}}\right), \mathrm{O}_{2}$ saturation $\left(\mathrm{SpO}_{2} \%\right)$, and $\mathrm{T}$ were also measured. D significantly reduced $\mathrm{T}$ compared to no stimulation for medium and high amplitudes. PRN also reduced T, without a difference between D and PRN. Furthermore, both stimulations significantly reduced $\mathrm{T}_{\mathrm{S}}$ with no significant differences between the respective stimulations. However, there was no effect of $\mathrm{D}$ or $\mathrm{PRN}$ on $\mathrm{SpO}_{2} \%$. The lack of differences between D and PRN led to an additional series of experiment comparing the same $\mathrm{D}$ to a band-limited white noise (WN) signal in young rats. Both $\mathrm{D}$ and $\mathrm{WN}$ were shown to significantly reduce T, with $\mathrm{D}$ showing statistical superiority in reduction of apnea. We further speculate that both deterministic and stochastic mechanical stimulations induce some form of mechanotransduction which is responsible for their efficacy, and our findings suggest that mechanical stimulation may be effective in treating AOP.
\end{abstract}

Keywords Premature infant $\cdot$ Mouse $\cdot$ Rat $\cdot$ Breathing $\cdot$ Mechanotransduction

\section{Introduction}

Approximately 15 million infants are born prematurely worldwide every year, and this rate continues to rise $[1,2]$. Complications associated with preterm birth constitute the single largest cause of death for infants under the age of five $[1,2]$. One of the most notorious causes of morbidity

Béla Suki

bsuki@bu.edu

1 Department of Biomedical Engineering, Boston University, 44 Cummington Mall, Boston, MA 02215, USA

2 Department of Emergency Medicine, Beth Israel Deaconess Medical Center, Harvard Medical School, Boston, MA, USA and mortality amongst preterm infants is their tendency for irregular breathing and apnea of prematurity (AOP) [3]. This variability in breathing may occur because of the infants' underdeveloped peripheral and central chemoreceptors, which can cause unstable respiratory responses [3-5]. Currently, the main form of treatment available for AOP is caffeine, which is used to stimulate the respiratory system [6-8]. Caffeine's long-term effects on the body remain controversial; studies have shown that caffeine may damage cerebral blood flow and retard infant growth, as well as a variety of other detrimental effects [9-12]. Due to these negative effects, there is an urgent need for a non-damaging and non-invasive treatment for AOP.

Several studies have focused on the utilization of mechanical stimulation to alleviate pathophysiological or medical 
conditions. For example, Priplata et al. [13] applied mechanical stimulation to stabilize motion by applying a low-level stochastic stimulus to an adult's insoles and showed that the stimulation can significantly assist with stability and movement without major discomfort. Similar applications have been developed to treat AOP, such as a vibrating mattress that produces subthreshold whole body stimulations [14-16]. The mattress has also been shown to reduce irritability in opioid-exposed infants [17]. The explanation behind the success of these mechanical stimulation-based approaches had been tied to the concept of stochastic resonance, which is the enhancement of an existing sub-threshold signal by the addition of a small amount of random noise [18].

However, the actual mechanism behind stochastic resonance in biological systems is still not fully understood. There may be other phenomena, unrelated to the stochastic nature of the vibrations, that can explain the effects of mechanical stimulations. Additionally, in a recent study, our laboratory showed that it is possible to reduce apnea in mice through the use of deterministic and local vibrations [19]. Previous studies have not fully investigated deterministic signals, so we aim to complement their findings. Accordingly, we hypothesized that the mechanism behind the reduction of apnea length is not a solely stochastic phenomenon, and suggest that deterministic, non-random mechanical stimulation could be equally as effective. To test this hypothesis, we aimed to investigate (1) if there is any difference between deterministic and nondeterministic stimulation on the length of apnea; and (2) whether the specific mechanical stimulation affects several additional physiological variables including carbon dioxide $\left(\mathrm{CO}_{2}\right)$ and oxygen saturation $\left(\mathrm{SpO}_{2}\right)$.

\section{Methods}

\subsection{Stimulation device}

Two vibrating mini motors (Adafruit 1201 Vibrating Mini Motor Disc, Adafruit, NYC) were attached to a rectangular rubber band. The vibrational motors were powered by a microcontroller (Arduino Mega 2560, Arduino, Somerville MA) connected to a computer with which stimulation patterns could be administered. For the experiment, the stimulation band was wrapped around the rodent's abdomen using Velcro on either side of the band. The dimensions of the stimulation band are similar between a rodent's abdomen and a neonate's wrist, allowing us to informally explore the physical design of our device. A human-scale prototype of this device has been developed for potential use in preterm infants, shown in Fig. 1 on a model of an infant's wrist (circumference $10 \mathrm{~cm}$ ). The prototype is 3D printed (MakerBot Replicator 2, Maker Filament PLA, Phil'a Mint Green, Burlington, WI) in two units, one that sits on top of the wrist

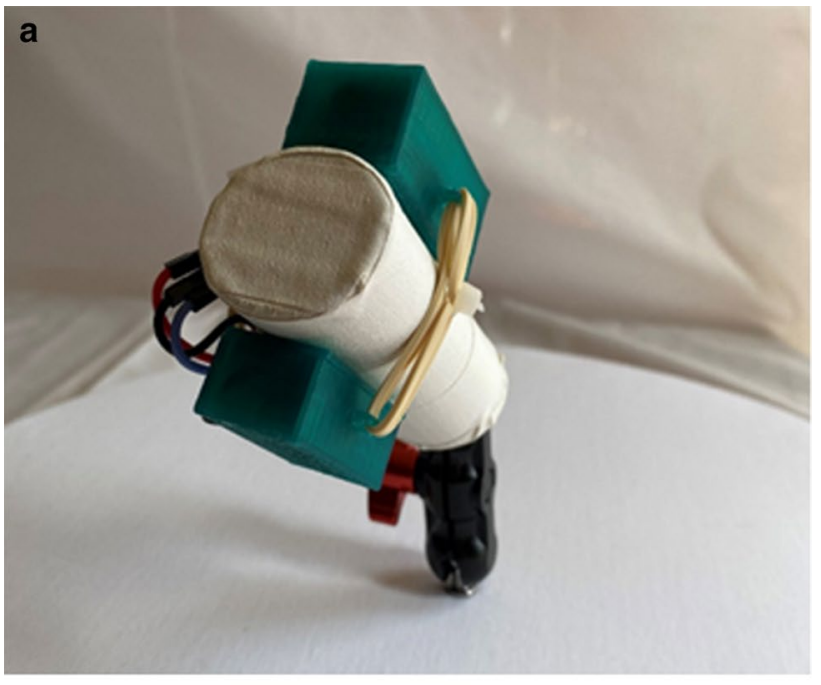

b
Casing Unit \# 1
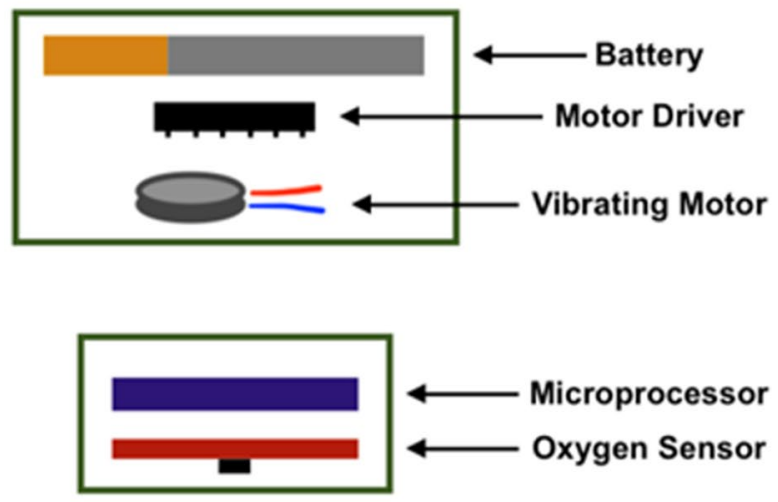

Casing Unit \# 2
Fig. 1 a A scaled-up prototype version of device designed for infants. The core of the device is 2 3D-printed plastic frames, one sits on top of the wrist, one below. The units close in a snap-fit design and have attachments on both sides of the casing to ensure secure connection around the infants wrist. b Schematic showing the inside components of each box. Unit 1 contains a rechargeable LiPo battery, a motor driver, and a mini motor. A support in the casing holds the motor in place. Unit \#2 contains a pulse oximeter sensor and a microprocessor

and one that sits below. This makes the device less bulky for a small infant. Unit \#1 measures $2 \mathrm{~cm} \times 2.5 \mathrm{~cm} \times 4 \mathrm{~cm}$ and contains a rechargeable LiPo battery (Adafruit 2750, Adafruit, NYC), a motor driver (ULN2003), and a mini motor (Adafruit 1201, Adafruit, NYC). Unit \#2 measures $1.5 \mathrm{~cm} \times 2.25 \mathrm{~cm} \times 3.5 \mathrm{~cm}$ and contains a pulse oximeter sensor (SEN-15219, Sparkfun) and a microprocessor (Adafruit 4600 QTPY, Adafruit, NYC). These dimensions were chosen in compliance with recommendations from the Neonatal Respiratory and Clinical Epidemiology Research Group (University Children's Hospital Basel, Switzerland). A gap in the casing allows the oxygen sensor to directly 
contact the skin. Both casing units open and close using a custom snap-fit design. Attachment points on either side of the casing units allow for an elastic bracelet to connect them, holding them securely on an infant's wrist.

\subsection{Experimental design and objectives}

All experiments were approved by the Institutional Animal Care and Use Committee of Boston University. A total of 5 mice (C57BL/6, male, 8-10 weeks) and 9 rats (Sprague Dawley, male, 40-75 days) were used in the studies. The experiments were divided into four subsets, each with a different objective.

(1) In the first subset $(\mathrm{N}=3$, mice), the sole objective was to optimize a deterministic square wave signal (D) in both voltage controlling vibration amplitude and frequency based on the time (T) to first breath after stopping the ventilator. No additional physiological measurements were done during these experiments. The frequency and amplitude of the signal were adjusted by controlling the pulse width modulation through its period and duty cycle. A total of six combinations of voltages $(1,1.5$, and $3 \mathrm{~V})$ and frequencies $(25 \mathrm{~Hz}$ and $50 \mathrm{~Hz}$ ) were tested, with four different settings (2 voltages and 2 frequencies) tested per mouse. At least 15 trials with every combination of voltages and frequencies were performed with each stimulation pattern being tested on two mice.

(2) The objective of the second subset ( $\mathrm{N}=2$, mice) was to compare the performance of the optimal $\mathrm{D}$ from subset \#1 with a pseudorandom noise (PRN) signal, and to study the effects of each signal on physiological measurements (see below). The stimulation time series was then exported and coded into the Arduino microcontroller. Our noise signal was originally intended to be a sum of sinusoids between 35 and $65 \mathrm{~Hz}$ in $1 \mathrm{~Hz}$ increments with equal amplitudes and random phases to match D. However, technical limitation in the delivery of the analog signal directly from our microcontroller catalyzed the formation of a very high frequency signal that is pseudorandom, with a mean amplitude of $1.5 \mathrm{~V}$. The Supplemental Fig. S1 compares these two signals in the time domain.

(3) The objective of the third subset $(\mathrm{N}=3$, rats $)$ was to test two additional non-random, low frequency stimulations that differ from the original deterministic stimulation in waveform and frequency content. We created two deterministic signals using our microcontroller, which we designated as light repetitive pulse (LRP) and strong repetitive pulse (SRP). In the neonatal intensive care unit (NICU), a nurse would stimulate an apneic infant by rubbing their hands or feet [20]. Previous work has reported comparable results between the mechanical stimulation delivered by a nurse and mechanical stimulation delivered by a computer, finding them to be comparable [15]. Accordingly, we designed our LRP and SRP as an automated alternative to a nurse's hand stimulation, to be delivered in response to detected apnea events. Both were delivered via the vibrating motors powered by $3.3 \mathrm{~V}$. LRP consists of a $250 \mathrm{~ms}$ pulse width, followed by a latent period of $750 \mathrm{~ms}$. SRP consists of a $500 \mathrm{~ms}$ pulse width, followed by a latent period of $500 \mathrm{~ms}$. The LRP can be considered as a periodic signal with an average amplitude of $0.825 \mathrm{~V}$ and frequency of $1 \mathrm{~Hz}$ while the SRP can be considered as a $1.655 \mathrm{~V}$ average amplitude signal with a frequency of $1 \mathrm{~Hz}$. This difference mimics an increase in the intensity and duty cycle of mechanical stimulation delivered by a NICU nurse. Supplemental Figure S2 compares these signals in the time domain. The power spectrum of the repetitive pulse signals, obtained by using a fast Fourier transform-based spectrogram, was also confirmed to be different from our original deterministic signal as shown in Supplemental Figure S3. Each experiment consisted of an average of 21 cycles of alternating control and LRP, and another 21 cycles of control followed by SRP.

(4) The objectives of the fourth subset $(\mathrm{N}=6$, rats) were to further validate our previous results by testing our device on another species, as well as to compare our deterministic stimulation to a stochastic, band-limited white noise (WN) signal with respect to T. The WN was crleated using a random number generator on the microcontroller that sent a digital signal to a digital-toanalogue converter (DAC) (MCP4725 Breakout Board, Adafruit, NYC). The DAC was connected to an operational amplifier (LM741, Texas Instruments, Dallas, Texas) that sent our desired signal to the mini motors. The signal produced was confirmed to be white noise using the spectrogram method. The power-spectra of PRN and WN are compared in Supplemental Fig. S4.

\subsection{Rodent Ventilation}

The experimental design closely followed the one described previously [19]. Briefly, mice (weight $=31.2 \pm 3.9 \mathrm{~g}$, Charles River) and rats (rats: weight $=219 \pm 33 \mathrm{~g}$, Charles River) were anesthetized intraperitoneally (mice: $0.1 \mathrm{ml} / 20 \mathrm{~g}$ Ketamine-Xylazine cocktail, Ketamine $87.5 \mathrm{mg} / \mathrm{kg}$, Xylazine $12.5 \mathrm{mg} / \mathrm{kg}$; rats: $0.4 \mathrm{ml} / 100 \mathrm{~g}$ Ketamine-Xylazine cocktail, Ketamine $100 \mathrm{mg} / \mathrm{kg}$ Xylazine $20 \mathrm{mg} / \mathrm{kg}$ ) and kept under mild anesthesia with additional doses of $0.05 \mathrm{ml} / 20 \mathrm{~g}$ Ketamine for mice and $0.25 \mathrm{ml} / 100 \mathrm{~g}$ of rat-cocktail as needed. Mice and rats were tracheostomized, and placed on a rodent mechanical ventilator (flexiVent Legacy (mice) and flexiVent 
FX module 4 (rats), Scireq Inc., Montreal) by connecting the tracheostomy tube to the ventilator. For rats, the FlexiVent module was capable of recording and displaying pressures directly from the tracheostomy tube. For mice, a separate pressure transducer (World Precision Instruments (WPI), 07B PNEU05) was attached to a side port of the tracheostomy tube to measure airway pressure during ventilation. In the second set of experiments, which compared D to PRN, a capnometer (MicroCapStar End-Tidal CO2 Analyzer, WPI) was connected to the tracheostomy tube through a t-tubing to measure $\mathrm{CO}_{2} \%$, and a pulse oximeter (MouseSTAT Jr. Rodent Pulse Oximeter, Kent Scientific) was placed on the paw of the mouse to measure $\mathrm{SpO}_{2} \%$.

The mice were ventilated with a tidal volume of $8 \mathrm{ml} / \mathrm{kg}$ at a rate of 180 or 200 breaths per minute superimposed on a positive end-expiratory pressure (PEEP) of $3 \mathrm{cmH}_{2} \mathrm{O}$. A stabilization phase followed before the onset of the actual experiment in order for the mouse to acclimate to the ventilator. Induction of apnea was achieved using cycles consisting of a $20 \mathrm{~s}$ ventilation period, followed by cessation of ventilation. With each ventilation pause, a selected stimulation was initiated or not used in the case of a control measurement. This is in line with current clinical practice standards, wherein apnea treatment begins soon after an apnea event is detected $[15,21]$. Once a few breaths were detected by the pressure transducer, ventilation was resumed, and the cycle was repeated with a different stimulation. For the deterministic signal optimization experiments, the order of stimulation type was randomized. This ensured that the set of different stimulations were carried out in relatively the same time period and the same number of times, but in random order. In total, 100 trials were performed with each stimulation type using 3 separate mice in this part of the experiment. For comparing D to PRN, the experimental design was modified so that the mouse received either PRN stimulation, D stimulation, or control. In addition, a control cycle followed both PRN stimulation and D stimulation to remove any potential bias or long-lasting effects of stimulation. A total of 80 trials were done for each stimulation type (PRN and D) and 160 trials were done for the control group using two separate mice.

The voltage signal from the transducer was amplified (WPI, Model TBM4-F), digitized (WPI, DataTrax), and displayed on a computer (WPI, Quad 16-EFA-400), while the capnometer voltage output was directly digitized. Once the experiment was completed, the two voltage time series were converted to gauge pressures. Since the pulse oximeter was unable to output the data, the $\mathrm{SpO} 2$ values were recorded manually at the onset of the first breath.

The rats were ventilated with a tidal volume of $20 \mathrm{ml} /$ $\mathrm{kg}$ at a rate of 120 breaths per minute superimposed on a PEEP of $3 \mathrm{cmH}_{2} \mathrm{O}$. A stabilization phase of $5 \mathrm{~min}$ followed before the onset of the actual experiment to ensure adequate adjustment of the animal to the ventilator as well as adequate anesthesia response. The rats' breathing was tracked in realtime using the pressure reading on the FlexiVent software. Rats received $30 \mathrm{~s}$ of ventilation, followed by a pause and induction of apnea. The cessation of ventilation was accompanied by activation of the motors in the cases of LRP, SRP, $\mathrm{D}$ and WN stimulations, or no activation in the case of control. In the first rat group $(\mathrm{N}=3)$, rats went through an average of 21 cycles of control followed by LRP, and then 21 cycles of control followed by SRP. A total of 63 cycles in each stimulation group and 126 cycles of control were in this set of experiments. For the last set of experiments testing deterministic and band-limited white noise stimulations, the order of stimulations was kept constant for all rat experiments and always began with control, followed by $\mathrm{D}$, followed by WN. In total, 6 rats were tested, with an average of 42.5 cycles per experiment and a minimum of 38 cycles per experiment. Experiments were terminated after 45 cycles, or sooner if inadequate anesthesia response was noted.

\subsection{Data analysis}

For each experiment, the following parameters were obtained for every breath:

1. T: The time to first breath following ventilation pause.

2. $\mathrm{T}_{\mathrm{s}}$ : Carbon dioxide stabilization time. After the first breath, the $\mathrm{CO}_{2}$ level dropped and remained erratic for several seconds before eventually stabilizing and returning to the previous stable state. The stabilization time was defined as the time needed for $\mathrm{CO}_{2}$ to return to a peak exhalation value that is within 0.4 percent of the $\mathrm{CO}_{2}$ level at first breath.

3. $\mathrm{CO}_{2}: \mathrm{CO}_{2}$ concentration at first breath.

4. $\mathrm{SpO}_{2} \%$ : Percent oxygen saturation level at first breath.

$\mathrm{T}_{\mathrm{s}}, \mathrm{SpO}_{2}$, and $\mathrm{CO}_{2}$ variables were collected only when comparing D to PRN.

Figure 2 shows an example of a cycle of experimental raw data. Figure $2 \mathrm{a}$ is generated from the pressure signal of the ventilated mouse. First breaths were established each cycle by detecting a pressure drop greater than $0.21 \mathrm{cmH}_{2} \mathrm{O}$. Pairing the capnometer with the ventilator and pressure transducer offered the additional advantage of confirming the first breath location by matching it to the location of the first peak of the capnometer reading after cessation of ventilation (shown in Fig. 2b). A constant lag of $1.3 \mathrm{~s}$ between the capnometer and pressure readings persisted throughout the experiments and was corrected in the analysis. Connecting the capnometer to the ventilator led to the formation of a system open to atmosphere for the brief period of time between the mouse's first independent breath and the return of ventilation. Therefore, we see a slight downwards sloping 

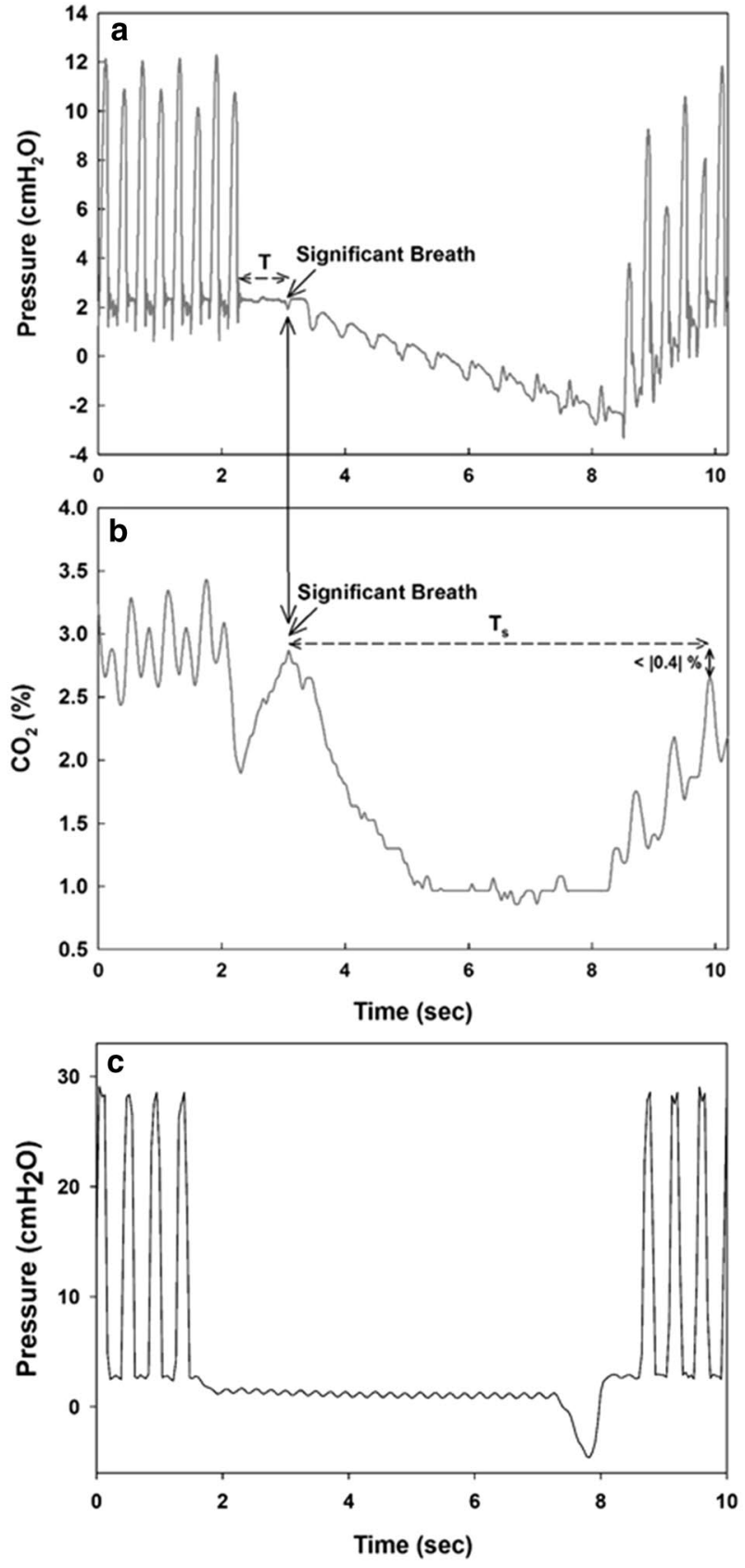

Fig. 2 Data collection and analysis. An example of a mouse ventilation cycle with measured $\mathbf{a}$ pressure and $\mathbf{b} \mathrm{CO}_{2} \%$ data with respect to time. After the ventilation is paused, a significant breath is detected from the pressure signal based on a fixed threshold of $0.21 \mathrm{~cm} \mathrm{H}_{2} \mathrm{O}$ pressure drop. This is also validated from the first $\mathrm{CO}_{2} \%$ peak following ventilation pause. Having located the significant breath, the time to breathe after pausing ventilation $(\mathrm{T})$ and the $\mathrm{CO}_{2} \%$ stabilization time $\left(\mathrm{T}_{\mathrm{s}}\right)$, defined as the time for the $\mathrm{CO}_{2} \%$ peak to reach $\pm 0.4 \%$ difference compared to the peak of the significant breath's peak. c An example of a ventilation cycle in a rat experiment, where no capnometer is attached and therefore the pressure remains flat from the end of ventilation to the onset of voluntary breathing. Breaths were detected using a threshold of $0.84 \mathrm{cmH}_{2} \mathrm{O}$ pressure drop of the pressure signal after T. Nevertheless, this still allowed us to reliably detect the mouse's first breath. The capnometer was utilized to make two other measurements. The first was the concentration of $\mathrm{CO}_{2}\left(\% \mathrm{CO}_{2}\right)$ at the time of first breath as read by the capnometer. The second measurement was the time for stabilization of the $\mathrm{CO}_{2}$ levels $\left(\mathrm{T}_{\mathrm{s}}\right)$. As shown in Fig. 2b, $\mathrm{T}_{\mathrm{s}}$ is defined as the time for the $\mathrm{CO}_{2}$ to return to within $0.4 \%$ of the levels at first breath.

Figure $2 \mathrm{c}$ shows an example of a ventilation cycle in a rat. No capnometer was used for the rat experiments and therefore the pressure curve remains flat from cessation of ventilation until the first voluntary breath. First breaths were established each cycle by detecting a pressure drop greater than $0.84 \mathrm{cmH}_{2} \mathrm{O}$. Rat experiments provided the added benefit of directly visualizing thoracic movement with attempted breaths; once ventilation was stopped, each rat was observed to confirm pressure readings.

During the long experiments, the ventilated rodents occasionally received additional doses of anesthesia. Hence, the raw times to first breath could vary over the course of an experiment, and between animals. In order to combine results from multiple experiments, the data from each experiment was normalized to the median of its control. Median normalization was used due to the skewness of the distribution, which also maintained consistency with our previous study [19]. The combined normalized data from every experimental setting was then tested for normality, and compared between the stimulation types using the nonparametric Friedman test followed by Bonferroni pairwise comparison. Statistical analyses to test for normality were done using SigmaPlot (Systat Software, Inc.) and all Friedman and Bonferroni tests were carried out in Matlab (Mathworks, Natick, MA). A P value less than 0.05 was considered to be significant.

\section{Results}

\subsection{Deterministic signal optimization}

Figure 3 summarizes the results from the deterministic signal optimization experiments. There was a statistically significant dependence on the voltage used to vibrate the mini motors. However, apnea length did not depend on frequency at any voltage suggesting a lack of frequency dependence for the experimentally-tested range. After normalization of $\mathrm{T}\left(\mathrm{T}_{\mathrm{n}}\right)$, it was noted that both $1.5 \mathrm{~V}$ and $3 \mathrm{~V}$ stimulations produced significant reductions in $\mathrm{T}_{\mathrm{n}}$ as compared to control. $\mathrm{T}_{\mathrm{n}}$ was reduced by $>16 \%$ using the $1.5 \mathrm{~V}$ stimulations $\left(P<10^{-7}\right)$ and reduced by $>8 \%$ using the $3 \mathrm{~V}, 25 \mathrm{~Hz}$ stimulation $(P<0.05)$. The $3 \mathrm{~V}, 50 \mathrm{~Hz}$ did not produce a statistical reduction in $\mathrm{T}_{\mathrm{n}}$ as compared to control $(P=0.113)$. The $1.5 \mathrm{~V}$ stimulations were statistically superior to the $3 \mathrm{~V}$ 


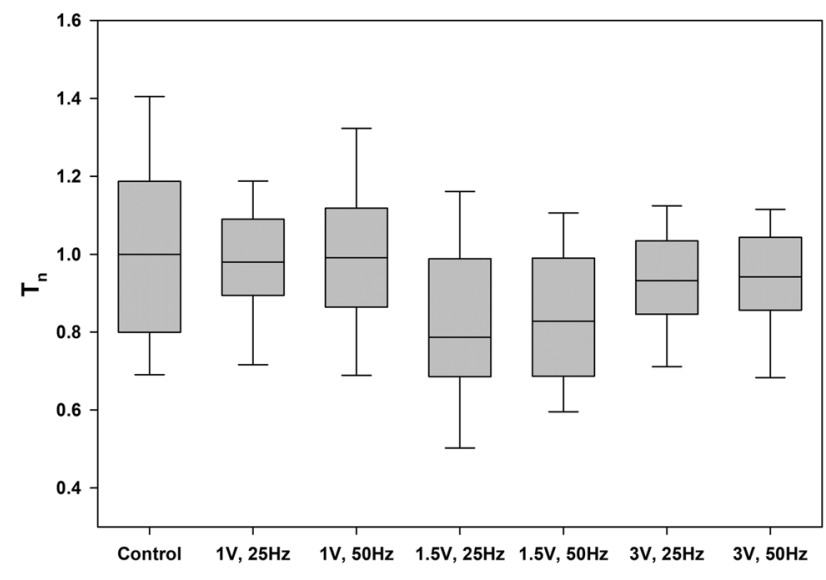

Fig. 3 Normalized time to first breath $\left(\mathrm{T}_{\mathrm{n}}\right)$ as affected by stimulations of different frequency and voltage combinations. The data are comprised of $\mathrm{N}=3$ mice, and a total of $\mathrm{n}=150$ cycles for each stimulation type. $\mathrm{T}_{\mathrm{n}}$ was normalized to the median of the control group, and significance was determined using ANOVA after normalization. No differences were detected between two frequencies at any given amplitude

stimulations at both frequencies $(P<0.05)$. The $1 \mathrm{~V}$ stimulation did not significantly reduce apnea length and was eventually deemed ineffective. A follow-up Friedman multiple comparison showed that all groups were different $\left(P<10^{-8}\right)$ with the $1.5 \mathrm{~V}$ stimulation being the most successful. On the other hand, independent of voltage, there was no effect of frequency in apnea length $(P=0.568)$.

\subsection{Comparison of the performance of $D$ and PRN}

Comparison of the performance of D to PRN demonstrates that although both $\mathrm{D}$ and PRN significantly decreased $\mathrm{T}$ by over $10 \%\left(P<10^{-5}\right)$, there was no difference between them. As shown in Fig. 4a, Friedman analysis of the normalized and combined data from the experiments confirmed these results with $\mathrm{T}_{\mathrm{n}}$ being $>14 \%$ shorter with both $\mathrm{D}$ and PRN stimulations $\left(P<10^{-4}\right)$. To eliminate any potential long-lasting effects of stimulation, we doubled the number of control cycles, placing one after both PRN cycles and D cycles. The control groups had a practically identical average $\mathrm{T}$ with no change in standard deviation and median $(P=0.723)$. Furthermore, our physiological measurements indicated that although both stimulations consistently drove the mice to breathe faster, ANOVA analysis showed there were no differences in $\mathrm{CO}_{2} \%$ or $\mathrm{SpO}_{2} \%$, at the time of first breath between any stimulation and the control groups $(P>0.4$ for all groups) (Table 1). This was also the case across all deterministic signals applied for mouse 3 (see Supplementary material, Table S1). Interestingly, however, Friedman analysis of the normalized $\mathrm{T}_{\mathrm{s}}\left(\mathrm{T}_{\mathrm{sn}}\right)$ showed PRN improving $\mathrm{T}_{\mathrm{sn}}$ by $14.4 \%\left(P<10^{-4}\right)$ and $\mathrm{D}$ improving $\mathrm{T}_{\mathrm{sn}}$ by $12.9 \%$
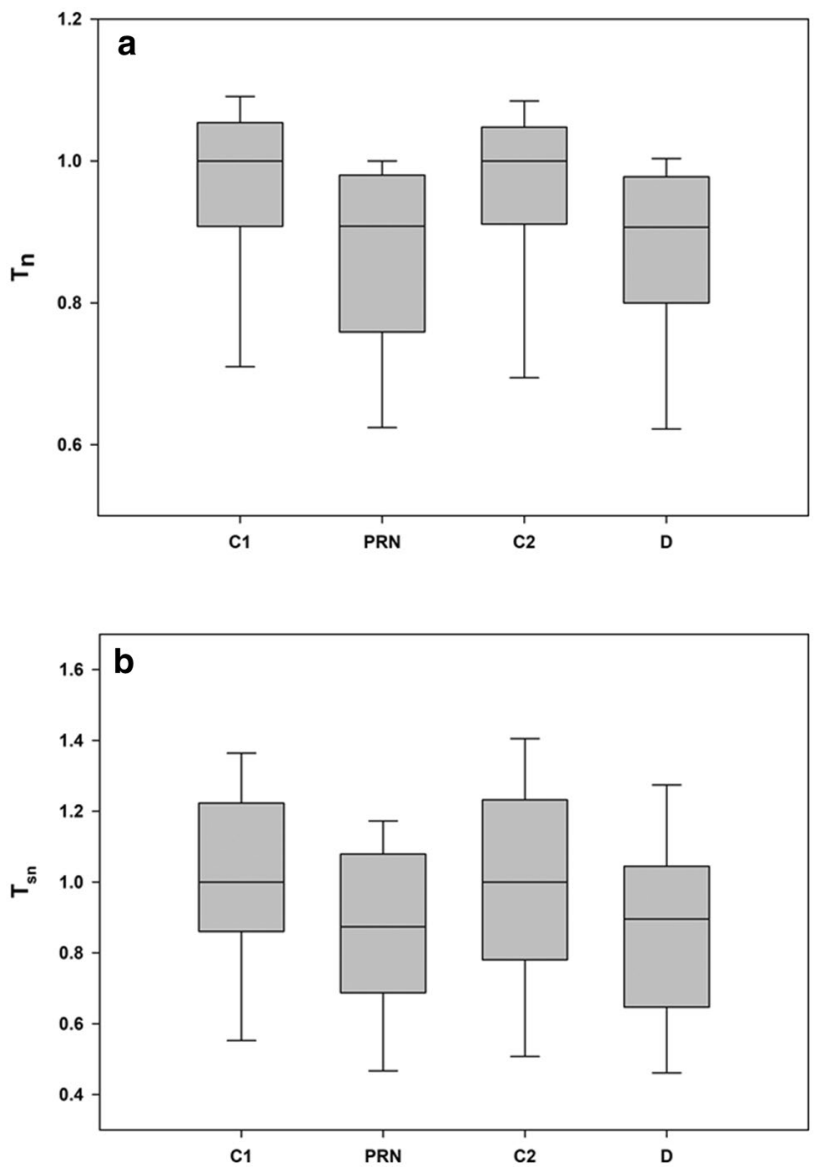

Fig. 4 a Effects of pseudorandom noise and deterministic (1.5 V, $50 \mathrm{~Hz})$ stimulation on the normalized time to first breath $\left(T_{n}\right)$ as compared to the no stimulation. $\mathrm{T}_{\mathrm{n}}$ was normalized to the median of the control group $(\mathrm{N}=2$ mice, $\mathrm{n}=80$ cycles). The two control groups $\left(C_{1}\right.$ and $\left.C_{2}\right)$ correspond to separate control cycles obtained after both PRN and D stimulations to check for fidelity and discount the possibility of a long-lasting effect of either stimulation. The separate control groups proved to be virtually identical $(P=0.987)$. b Normalized time for $\mathrm{CO}_{2}$ stabilization $\left(\mathrm{T}_{\mathrm{sn}}\right)$ is affected by both the pseudorandom noise and the deterministic $(1.5 \mathrm{~V}, 50 \mathrm{~Hz})$ stimulation compared to no stimulation. $\mathrm{T}_{\mathrm{sn}}$ was normalized to the median of the control group ( $\mathrm{N}=2$ mice, $\mathrm{n}=80$ cycles). The two control groups correspond to separate control cycles obtained after both PRN and D stimulations to check for fidelity and discount the possibility of a long-lasting effect of either stimulation. The separate control groups proved to be virtually identical $(P=0.987)$

$\left(P<10^{-4}\right)$ as compared to control. There was no significant difference between PRN and D with respect to their effect on $\mathrm{T}_{\mathrm{sn}}$. The two control groups were also compared with practically identical results $(P=1.00)$. A summary of the $\mathrm{T}_{\mathrm{sn}}$ results is shown in Fig. $4 \mathrm{~b}$.

\subsection{The effects of LRP and SRP}

Figure 5 describes the results obtained from the rat experiments investigating the effects of LRP and SRP on $T_{n}$. Using 
Table 1 Comparison of physiological measurements among control, deterministic and pseudorandom noise

\begin{tabular}{|c|c|c|c|c|c|c|c|}
\hline \multirow[t]{2}{*}{ Mouse } & \multirow[t]{2}{*}{ Cycles } & \multicolumn{3}{|l|}{$\mathrm{CO}_{2} \%$} & \multicolumn{3}{|l|}{$\mathrm{SpO}_{2} \%$} \\
\hline & & C & D & PRN & $\mathrm{C}$ & $\mathrm{D}$ & PRN \\
\hline 4 & 40 & $2.95 \pm 0.18$ & $2.95 \pm 0.23$ & $3.00 \pm 0.22$ & $79.7 \pm 1.0$ & $80.9 \pm 2.2$ & $80.2 \pm 0.9$ \\
\hline 5 & 40 & $2.83 \pm 0.24$ & $2.80 \pm 0.22$ & $2.80 \pm 0.22$ & $95.8 \pm 1.7$ & $95.7 \pm 1.9$ & $94.8 \pm 1.6$ \\
\hline
\end{tabular}

ANOVA analysis indicated there was no significant difference in $\mathrm{CO}_{2} \%$ or $\mathrm{SpO}_{2} \%$ among the groups

C: Control. D: Deterministic square signal, V =1.5 V, F=50 Hz. PRN: Pseudorandom noise

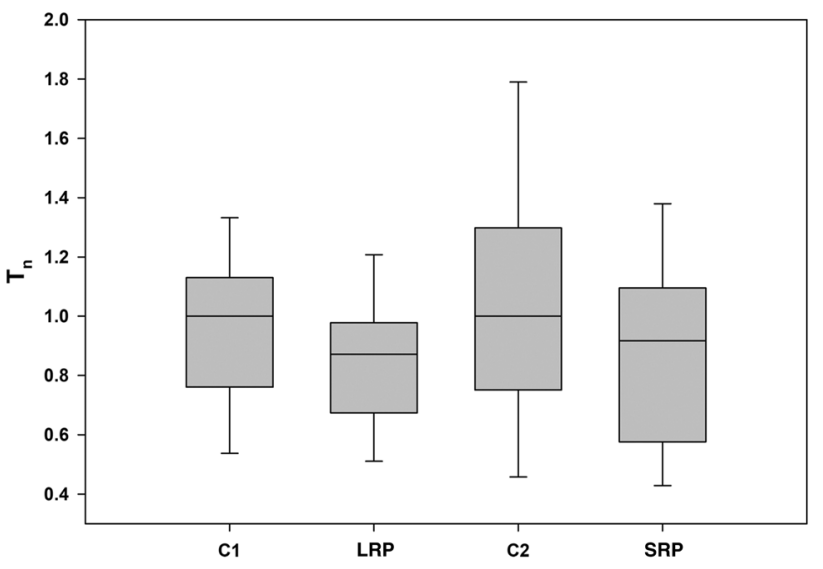

Fig. 5 Normalized time to first breath $\left(T_{n}\right)$ is affected by low-frequency repetitive pulse of both light and strong quality. $T_{n}$ was normalized to the median of the control group of the stimulation. The two control groups $\left(\mathrm{C}_{1}\right.$ and $\left.\mathrm{C}_{2}\right)$ are separate as each came before either LRP or SRP to discount any long-lasting effects of either stimulation. To ensure fidelity, the control groups were compared to one another to confirm lack of significant differences $(\mathrm{N}=3$ rats, $\mathrm{n}=63$ cycles)

Friedman and Bonferonni tests, both LRP and SRP stimulations were found to successfully reduce apnea as compared to control by an average of $11.2 \%(P<0.05)$ and $17.3 \%$ $(P<0.05)$, respectively. LRP and SRP were compared to one another and no significant difference was detected $(P=1.00)$. Additionally, the two control groups were compared to one another to ensure validity of data, and no difference was noted. No significant reduction in $\mathrm{T}$ was noted for individual rats with either LRP or SRP as compared to control, but a trend for more stable and faster breathing was certainly noted as seen by the significance of the cumulative normalized data.

\subsection{The effects of WN and D}

Analysis of the effects of $\mathrm{WN}$ and D stimulations on $\mathrm{T}$ has further supported the ability of mechanical stimulation to reduce apnea length. The results of the rat experiments comparing the effects of $\mathrm{D}$ and $\mathrm{WN}$ stimulation on normalized $\mathrm{T}$ are summarized in Fig. 6. Friedman analysis of the normalized time to first breath confirmed that D and WN stimulations reduced $\mathrm{T}_{\mathrm{n}}$ by $10.4 \%$ and $6.9 \%$ respectively compared

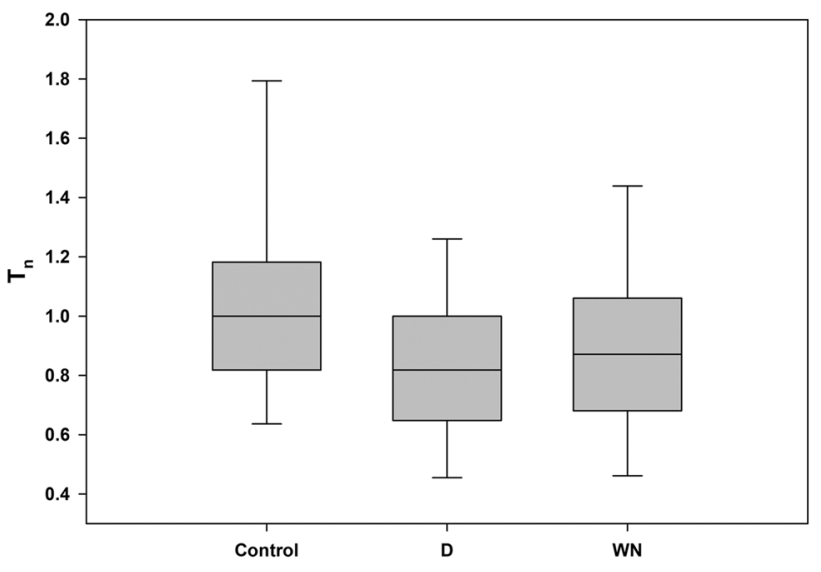

Fig. 6 Normalized time to first breath $\left(\mathrm{T}_{\mathrm{n}}\right)$ is consistently reduced in ventilated rats using mechanical stimulation $(\mathrm{N}=6$ rats, $\mathrm{n}=255$ cycles). Both $\mathrm{D}(1.5 \mathrm{~V}, 50 \mathrm{~Hz})$ and $\mathrm{WN}$ are superior to control with $P<10^{-2}$ when either is compared to control. A significant difference was traced between D and WN $(P<0.0001)$, with deterministic stimulation improving $\mathrm{T}$ by an average of $10.4 \%$ and $\mathrm{WN}$ improving $\mathrm{T}$ by an average of $6.9 \%$

to control $\left(P<10^{-4}\right)$. Friedman comparison of D and WN stimulations for all 6 experiments showed $\mathrm{D}$ as significantly superior to band limited $\mathrm{WN}$ at reducing $\mathrm{T}_{\mathrm{n}}(P<0.01)$.

\section{Discussion}

This study has multiple implications. First, we verified the findings of our previous experiment showing that local mechanical stimulation can help alleviate symptoms of apnea and reduce apnea length in an intensity-dependent manner [19]. We were able to show the consistency with which mechanical stimulation can reduce apnea across multiple species. Additionally, we have also shown that (1) the underlying mechanism is not necessarily stochastic in nature, (2) mechanical stimulation reduces $T_{s}$, and (3) repetitive low frequency mechanical stimulation can also be used for reduction of apnea.

The results show that the relationship between stimulation amplitude and apnea relief is threshold-dependent (Fig. 3). We found that $1.5 \mathrm{~V}$ stimulation produces the most successful result in reducing apnea length, and that doubling the amplitude almost halves the effectiveness. 
Reducing the amplitude to $1 \mathrm{~V}$ stimulation and approaching the subthreshold region (as indicated by the experimenters feeling the stimulation approximately half of the time) resulted in no significant effect compared to control and was essentially ineffective stimulation. This suggests that there is an optimal level of mechanical vibrations on the abdomen at which the reduction of AOP is maximized. Further experimentation may be able to pinpoint an exact optimum as was attempted in previous studies [22-24]. Interestingly, our data shows that there is no optimal frequency within the range studied here. Nevertheless, the successful reduction in apnea length by these deterministic signal stimulations hints at the possibility that the underlying mechanism is not entirely stochastic and may have other mechanical influences. Stochastic resonance signals involve white noise that contains a wide spectrum of frequencies with random phases [18]. While the success of many performance-enhancing and stabilizing devices, such as the AOP vibrating mattress described by Bloch-Salisbury et al. [14], has been mostly attributed to a stochastic mechanism, our results provide evidence that other mechanisms can also contribute to the observed phenomena and elicit similar effects. These mechanisms may already be playing a role in the success of therapeutic modalities such as the vibrating mattress. The experiments reported here show that stochastic resonance is unlikely to be the mechanism that reduces the time to first breath. Indeed, the results in Figs. 4 and 6 demonstrate that while the pseudorandom and band-limited white noise stimulations reduced apnea length from control, they were not better than the deterministic stimulation. A pervious study by Paydarfar et al. [25] has attempted to find deterministic signals that produce matching results to a previously optimized stochastic signal in single giant squid axons. However, no generalized full body stimulation nor respiratory effects of deterministic signals have been previously reported in that study or elsewhere.

While the creation of the pseudorandom noise was affected by technical challenges, making it a non-stochastic signal, it is remarkably different from our deterministic signal (see supplemental Fig. S1). Supplemental Fig. S4 portrays the power spectra of one period of the WN and PRN stimulations and highlights the differences and similarities between them. The two signals span the same range of frequencies, although the PRN appears to have more power at higher frequencies. Additionally, our approximation can be seen as reliable since the results from our rat experiments showed an extremely similar reciprocity with regards to the effects of PRN and WN on T compared to control or deterministic. The consistency with which our results are reproduced with both mice and rats is encouraging in that our mechanical stimulation can reduce apnea length in a frequency-independent fashion. Although not yet tested in a preterm infant model, our device carries potential for the treatment of AOP.

Our results with the two repetitive pulse signals imply that a low-frequency signal may also be able to stabilize breathing patterns while conserving device battery power. This further supports the notion that the reduction in apneic intervals can be achieved with both mechanical and stochastic inputs. Moreover, repetitive pulse stimulation may have an advantage over delivering a constant stimulus due to the physiology of action potential initiation in neurons that govern the breathing mechanism in infants [26]. Crago at el. showed that delivering a repetitive stimulus with magnitude greater than the action potential threshold, and at a rate less than or equal to the native neuronal firing rate, leads to partial rate summation and amplification of the original signal [27]. This may play a role in the success of the repetitive pulse stimulation and could constitute the electrophysiology basis of future research.

With respect to the actual mechanism, we note that in general, mechanical stimulation, known as mechanotransduction, can induce biochemical signaling in many cell types [27-29]. In fact, mechanical fluctuations can trigger different signaling pathways than regular deterministic mechanical signals $[29,30]$. Since a breath is initiated from the respiratory rhythm generator, a network of 5 groups of respiratory neurons that receive inputs from the periphery can be used to mimic neuronal discharge patterns [31]. Hence, the most likely possibility is that the stimulation induces mechanotransduction in the peripheral nerves related to respiratory control. Mechanical stimulation of nerve terminals has been found to induce action potentials [29]. While little is known about the actual mechanisms of mechanotransduction leading to action potentials, the cytoskeleton-including actin and microtubules-as well as mechanosensitive channels are involved in the mechanotransduction process [28]. It remains to be further investigated which neurons are involved and how the brain responds to such peripheral mechanical stimulation.

An important implication stemming from this study is the possibility that subthreshold stimulation may not be optimal for alleviating symptoms of AOP. Previous devices that are said to administer subthreshold stimulation, which is not to be felt by the infant, have been studied for their ability to help reduce instances and lengths of apnea [14]. In our study, however, we found that stimulation near the threshold of conscious detection was in fact much less effective in reducing apnea length than stronger suprathreshold stimulations. While we cannot extrapolate this finding in anesthetized mice to human infants, more research into the possible effects of slightly stronger mechanical stimulations on preterm infants with AOP is warranted. There remains the possibility that stronger mechanical stimulations could rouse an apneic infant to wakefulness, which may have unintended 
effects that should be considered in a risk-benefit analysis alongside the dangers of AOP.

It is important to note that we do not claim to make a comparison between the stochastic stimulation applied in previous work [32] and the one applied in our study. There are multiple methodological differences that preclude a direct comparison such as location of stimulation, magnitude, duration and detectability of the signal, as well as subjects used in the studies. Additionally, our stochastic approach remains in the realm of classical stochastic resonance, but future work may investigate other fields of noise effects [18]. We also recognize that a deterministic signal is not necessarily simpler in nature than a stochastic signal, yet we found it much more energy efficient to deliver using our small, wearable device. Finally, further investigation is warranted in order to extrapolate the reduction in $\mathrm{T}$ by deterministic stimuli observed in rodents to reducing AOP in human babies.

In addition to measuring apnea length, we also acquired several physiological variables which are routinely collected in a clinical setting. An interesting finding is the lack of differences in $\mathrm{CO}_{2}$ levels and oxygen saturation between the stimulation and control groups at the time of first breath. The lack of changes in these parameters, despite the mouse's tendency to breathe faster with stimulation, may be due to an enhanced gas mixing in the lung resulting from the mechanical vibrations. Since the mouse breathes faster with stimulation than without, but the oxygen saturation and $\mathrm{CO}_{2}$ levels at the time of first breath are the same, we can postulate that both $\mathrm{SpO}_{2}$ and $\mathrm{CO}_{2}$ levels simply change faster with than without stimulation. This is also supported by the finding that $\mathrm{CO}_{2}$ levels stabilize significantly faster with stimulation. The lack of significant differences between the pseudorandom noise and deterministic stimulations with regard to the stabilization time of $\mathrm{CO}_{2}$ also suggests that a purely mechanical mixing process is taking place.

This study has several important limitations. First, all experiments used adult mice and rats as an animal model for preterm infants. Adult rodents may be more responsive to stimulation than prematurely born ones. It is also possible that anesthesia may have an effect that makes the animal respond differently to the stimulation applied but conducting these experiments without anesthesia is not possible. Additionally, it remains unclear whether and to what extent our findings in rodents will translate to human subjects. Our rodent model also precludes characterizing long-term breathing pattern changes that may be occurring due to each stimulation. We also note the excitation signal supplied to the vibrational motors is being modulated in terms of frequency and amplitude as a caveat of the motors' eccentric rotating mass (ERM) properties. However, the properties of our signals allow us to safely conclude that this modulation does not affect the type of stimulation supplied; our deterministic signals are still deterministic and the noise signals are still stochastic or pseudorandom. Finally, all stimulations were applied onto the rodent's abdomen, which differs from the location of applied stimulus on an infant that would be on the ankle or wrist. Our previous study found no significant difference in a rodent's response to stimulation between the limb and the abdomen; therefore, we believe that our choice of stimulation placement does not significantly impact the results [19].

In conclusion, we have created a prototype mechanical stimulator that is portable, small in size, and able to be worn on an infant's wrist, as well as showed that this device is able to reduce apnea length in adult mouse and rat models of apnea. We also showed evidence that the mechanism behind the reduction of apnea length is not limited to stochastic resonance and argued that local mechanotransduction in peripheral nerves plays a nontrivial role. The prototype is ready for preclinical testing and has the potential to reduce AOP, which could be lifesaving for prematurely born infants.

Supplementary Information The online version contains supplementary material available at https://doi.org/10.1007/s13534-021-00203-x.

Acknowledgements The authors thank Dr. Sven Schulzke for guidance on designing the prototype. The authors also thank Sefik Evren Erdener and Kivilcim Kilic (Neurophotonics Center, Boston University) for help with the Capnometer and pulse oximeter measurements.

\section{Declarations}

Conflict of interest There is no conflict of interest for any authors.

\section{References}

1. Blencowe H, Cousens S, Oestergaard MZ, Chou D, Moller A-B, Narwal R, et al. National, regional, and worldwide estimates of preterm birth rates in the year 2010 with time trends since 1990 for selected countries: a systematic analysis and implications. Lancet. 2012;379:2162-72. https://doi.org/10.1016/S01406736(12)60820-4.

2. Lawn JE, Davidge R, Paul VK, von Xylander S, de Graft JJ, Costello A, et al. Born too soon: care for the preterm baby. Reprod Health. 2013;10(Suppl 1):S5. https://doi.org/10.1186/ 1742-4755-10-S1-S5.

3. Fyfe KL, Odoi A, Yiallourou SR, Wong FY, Walker AM, Horne RSC. Preterm infants exhibit greater variability in cerebrovascular control than term infants. Sleep. 2015;38:1411-21. https:// doi.org/10.5665/sleep.4980.

4. Frey U, Silverman M, Barabási AL, Suki B. Irregularities and power law distributions in the breathing pattern in preterm and term infants. J Appl Physiol. 1985;1998(85):789-97. https://doi. org/10.1152/jappl.1998.85.3.789.

5. Glass HC, Costarino AT, Stayer SA, Brett CM, Cladis F, Davis PJ. Outcomes for extremely premature infants. Anesth Analg. 2015;120:1337-51. https://doi.org/10.1213/ANE.0000000000 000705 . 
6. Zhao J, Gonzalez F, Mu D. Apnea of prematurity: from cause to treatment. Eur J Pediatr. 2011;170:1097-105. https://doi.org/10. 1007/s00431-011-1409-6.

7. Eichenwald EC, Committee on Fetus and Newborn, American Academy of Pediatrics. Apnea of prematurity. Pediatrics. 2016. https://doi.org/10.1542/peds.2015-3757.

8. Martin RJ, Abu-Shaweesh JM, Baird TM. Apnoea of prematurity. Paediatr Respir Rev. 2004;5(Suppl A):S377-382. https://doi.org/ 10.1016/s1526-0542(04)90067-x.

9. Hoecker C, Nelle M, Poeschl J, Beedgen B, Linderkamp O. Caffeine impairs cerebral and intestinal blood flow velocity in preterm infants. Pediatrics. 2002;109:784-7. https://doi.org/10.1542/peds. 109.5.784.

10. Ergenekon E, Dalgiç N, Aksoy E, Koç E, Atalay Y. Caffeine intoxication in a premature neonate. Paediatr Anaesth. 2001;11:737-9. https://doi.org/10.1046/j.1460-9592.2001.00753.x.

11. Anderson BJ, Gunn TR, Holford NH, Johnson R. Caffeine overdose in a premature infant: clinical course and pharmacokinetics. Anaesth Intensive Care. 1999;27:307-11. https://doi.org/10.1177/ $0310057 X 9902700316$.

12. Carnielli VP, Verlato G, Benini F, Rossi K, Cavedagni M, Filippone $\mathrm{M}$, et al. Metabolic and respiratory effects of theophylline in the preterm infant. Arch Dis Child Fetal Neonatal Ed. 2000;83:F39-43. https://doi.org/10.1136/fn.83.1.f39.

13. Priplata AA, Niemi JB, Harry JD, Lipsitz LA, Collins JJ. Vibrating insoles and balance control in elderly people. Lancet. 2003;362:1123-4. https://doi.org/10.1016/S0140-6736(03) 14470-4.

14. Bloch-Salisbury E, Indic P, Bednarek F, Paydarfar D. Stabilizing immature breathing patterns of preterm infants using stochastic mechanosensory stimulation. J Appl Physiol. 1985;2009(107):1017-27. https://doi.org/10.1152/japplphysiol. 00058.2009.

15. Pichardo R, Adam JS, Rosow E, Bronzino J, Eisenfeld L. Vibrotactile stimulation system to treat apnea of prematurity. Biomed Instrum Technol. 2003;37:34-40. https://doi.org/10.2345/08998205(2003)37[34:vsstta]2.0.co;2.

16. Smith VC, Kelty-Stephen D, Qureshi Ahmad M, Mao W, Cakert $\mathrm{K}$, Osborne J, et al. Stochastic resonance effects on apnea, bradycardia, and oxygenation: a randomized controlled trial. Pediatrics. 2015;136:e1561-1568. https://doi.org/10.1542/peds.2015-1334.

17. Zuzarte I, Indic P, Barton B, Paydarfar D, Bednarek F, BlochSalisbury E. Vibrotactile stimulation: a non-pharmacological intervention for opioid-exposed newborns. PLoS ONE. 2017;12: e0175981. https://doi.org/10.1371/journal.pone.0175981.

18. McDonnell MD, Abbott D. What is stochastic resonance? Definitions, misconceptions, debates, and its relevance to biology. PLoS Comput Biol. 2009;5: e1000348. https://doi.org/10.1371/journal. pcbi.1000348.

19. Bou Jawde S, Scheuermann A, Bartolák-Suki E, Suki B. The effect of mechanical or electrical stimulation on apnea length in mice. Biomed Eng Lett. 2018;8:329-35. https://doi.org/10.1007/ s13534-018-0076-1.
20. Suki B, Parameswaran H, Imsirovic J, Bartolák-Suki E. Regulatory roles of fluctuation-driven mechanotransduction in cell function. Physiology (Bethesda). 2016;31:346-58. https://doi.org/10. 1152/physiol.00051.2015.

21. Mohr MA, Vergales BD, Lee H, Clark MT, Lake DE, Mennen AC, et al. Very long apnea events in preterm infants. J Appl Physiol. 1985;2015(118):558-68. https://doi.org/10.1152/japplphysiol. 00144.2014.

22. Forger DB, Paydarfar D. Starting, stopping, and resetting biological oscillators: in search of optimum perturbations. J Theor Biol. 2004;230:521-32. https://doi.org/10.1016/j.jtbi.2004.04.043.

23. Forger DB, Paydarfar D, Clay JR. Optimal stimulus shapes for neuronal excitation. PLoS Comput Biol. 2011;7: e1002089. https://doi.org/10.1371/journal.pcbi.1002089.

24. Chang J, Paydarfar D. Optimizing stimulus waveforms for electroceuticals. Biol Cybern. 2019;113:191-9. https://doi.org/10.1007/ s00422-018-0774-x.

25. Paydarfar D, Forger DB, Clay JR. Noisy inputs and the induction of on-off switching behavior in a neuronal pacemaker. J Neurophysiol. 2006;96:3338-48. https://doi.org/10.1152/jn.00486.2006.

26. Bartolák-Suki E, Imsirovic J, Parameswaran H, Wellman TJ, Martinez N, Allen PG, et al. Fluctuation-driven mechanotransduction regulates mitochondrial-network structure and function. Nat Mater. 2015;14:1049-57. https://doi.org/10.1038/nmat4358.

27. Crago PE, Makowski NS. Alteration of neural action potential patterns by axonal stimulation: the importance of stimulus location. $\mathrm{J}$ Neural Eng. 2014;11: 056016. https://doi.org/10.1088/1741-2560/ 11/5/056016.

28. Manos T, Zeitler M, Tass PA. How stimulation frequency and intensity impact on the long-lasting effects of coordinated reset stimulation. PLoS Comput Biol. 2018;14: e1006113. https://doi. org/10.1371/journal.pcbi.1006113.

29. Lin Y-W, Cheng C-M, Leduc PR, Chen C-C. Understanding sensory nerve mechanotransduction through localized elastomeric matrix control. PLoS ONE. 2009;4: e4293. https://doi.org/10. 1371/journal.pone.0004293.

30. Martino F, Perestrelo AR, Vinarský V, Pagliari S, Forte G. Cellular mechanotransduction: from tension to function. Front Physiol. 2018;9:824. https://doi.org/10.3389/fphys.2018.00824.

31. Wang N. Review of cellular mechanotransduction. J Phys D Appl Phys. 2017;50:233002.

32. McDonnell MD, Ward LM. The benefits of noise in neural systems: bridging theory and experiment. Nat Rev Neurosci. 2011;12:415-25. https://doi.org/10.1038/nrn3061.

Publisher's Note Springer Nature remains neutral with regard to jurisdictional claims in published maps and institutional affiliations. 